\title{
COMPLICAÇÃO DA BANDA GÁSTRICA AJUSTÁVEL VIDEOLAPAROSCÓPICA PARA TRATAMENTO DA OBESIDADE MÓRBIDA: EXTRUSÃO DA BANDA
}

\author{
COMPLICATION OF ADJUSTABLE GASTRIC BANDAGE THROUGH \\ VIDEOLAPAROSCOPY FOR MORBID OBESITY: BAND EXTRUSION
}

\author{
Ana Maria Zuccaro, TCBC-RJ ${ }^{1}$ \\ Huang Ling Fang ${ }^{2}$
}

\section{INTRODUÇÃO}

A obesidade mórbida é um problema de saúde pública, com implicações médicas, sociais, psicológicas, econômicas e, principalmente, modificadoras da qualidade de vida. Observamos, nesses pacientes, incidência elevada de doenças como coronariopatia, hipertensão arterial, diabetes, dislipidemia, doença osteoarticular degenerativa, apnéia do sono, alguns tipos de tumores malignos e morte súbita.

Na última década, foram propostos novos modelos de tratamento cirúrgico para a obesidade através da redução do reservatório gástrico, a gastroplastia vertical, as técnicas disabsortivas como o by pass jejuno-ileal e a derivação bileo-pancreática, e as técnicas restritivas/disabsortivas, a gastroplastia vertical com by pass intestinal.

A banda gástrica ajustável de silicone por via vídeo-laparoscópica (BGAVL) é a cirurgia bariátrica mais utilizada na Europa ${ }^{1}$. É um procedimento seguro, sem mortalidade referida ao ato cirúrgico, porém, não é isento de complicações. Dentre os fatores relacionados com o insucesso da banda e necessidade de re-operação destaca-se a presença de hérnia hiatal ou dismotilidade esofagiana prévia ${ }^{2}$. As complicações pós-operatórias podem estar relacionadas com o porta-cath, o ato cirúrgico ou a banda gástrica. São descritos processos infecciosos com port ou com a banda. Dentre as complicações clínicas decorrentes da cirurgia observa-se a dilatação esofagiana e a dilatação precoce da bolsa gástrica ${ }^{3-5}$.

\section{RELATO DOS CASOS}

\section{Caso 1}

Paciente feminina, 44 anos, submetida a BGVL (banda gástrica de silicone BioEnterics ${ }^{\circledR}$ ), no HGI, há dezenove meses por obesidade mórbida com IMC de 52. Nos últimos três meses evoluiu com vômitos à ingesta de alimentos sólidos e disfagia intermitente. A endoscopia digestiva alta foi indicada pelo agravamento do quadro clínico nas duas semanas que antecederam o exame.

Realizamos a esofagogastroduodenoscopia (aparelho videoendoscópio Fujinon 201), sob sedação consciente com benzodiazepínico. Foi realizado monitorização da saturação de oxigênio e frequiência cardíaca através de oximetria capilar.

Ao exame endoscópico, à retroversão do aparelho, observamos a perfuração gástrica com a extrusão da banda na luz gástrica (Figura-1).

1. Mestre em Gastroenterologia; Chefe do Serviço de Endoscopia do Hospital Geral de Ipanema (HGI) e do Serviço de Endoscopia e Fisiologia Digestiva da Policlínica Geral do Rio de Janeiro (PGRJ)

2. Médica do Serviço de Endoscopia e Fisiologia Digestiva da Policlínica Geral do Rio de Janeiro (PGRJ) e Médica do Serviço de Coloproctologia do Hospital Universitário Clementino Fraga Filho da UFRJ

Trabalho realizado no Serviço de Endoscopia Digestiva do Hospital Geral de Ipanema (HGI) e no Serviço de Endoscopia e Fisiologia Digestiva da Policlínica Geral do Rio de Janeiro (PGRJ), Rio de Janeiro, RJ 


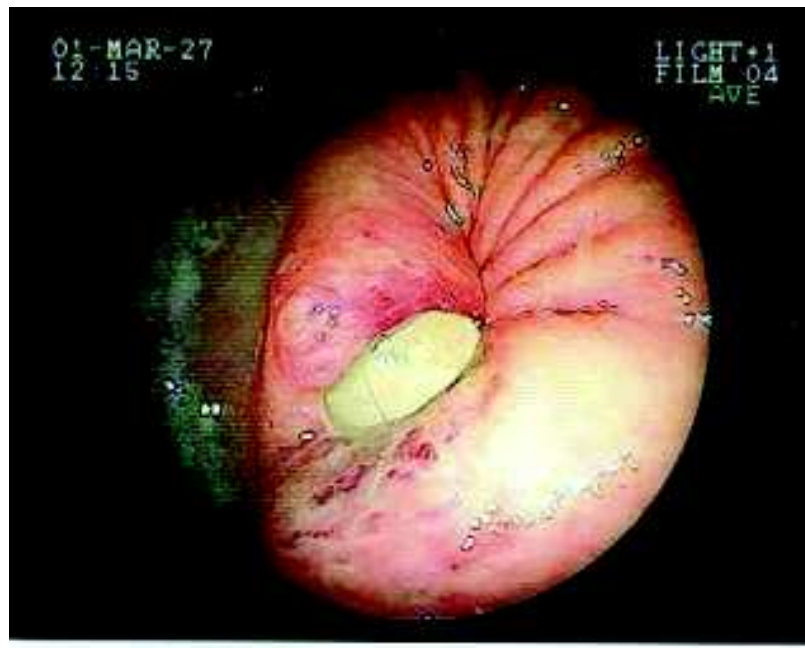

Figura 1 - Extrusão da banda gástrica ajustável: retroversão e rotação do aparelho; identificação da banda de silicone na luze do processo inflamatório adjacente (fotovideoendoscopia)

A banda gástrica foi retirada através de videolaparoscopia. Durante o ato cirúrgico não foi identificado o local da perfuração, sendo necessário a realização de videoendoscopia per-operatória com identificação endoscópica do local da perfuração, introdução de fio-guia através do canal de biópsia e visualização do fio-guia intra-abdominal.

\section{Caso 2}

Paciente feminina, 34 anos, no vigésimo quarto mês de pós-operatório de BGAVL realizada em outro estado (SP), procurou o Serviço de

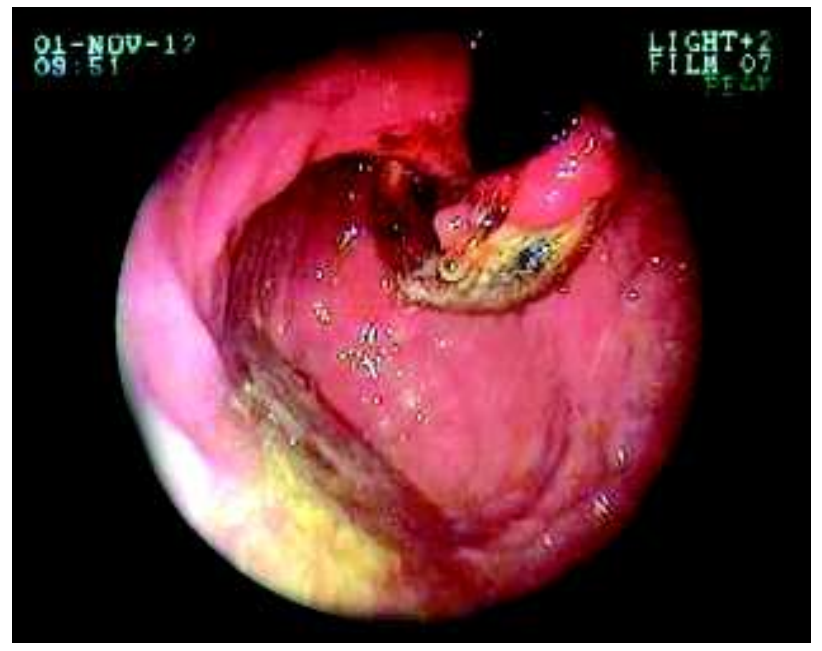

Figura 2 - Extrusão da banda gástrica ajustável: retroversão; identificação da BGAVL no fundo gástrico (fotovideoendoscopia)
Endoscopia e Fisiologia Digestiva da PGRJ relatando disfagia progressiva para sólidos e líquidos nos últimos dois meses e dor abdominal. Do mesmo modo que no caso anterior, a região epigástrica era extremamente dolorosa à palpação superficial e profunda, sem reação de defesa.

A endoscopia foi realizada dentro dos mesmos critérios referidos no caso anterior. A banda foi facilmente visualizada na luz gástrica à retroversão do aparelho. Neste caso, a banda apresentava uma maior extrusão e menor reação inflamatória (Figura-2).

Como no primeiro caso, optamos pela retirada cirúrgica da banda gástrica através do acesso videolaparoscópico sem conversão em outra técnica bariátrica. A endoscopia digestiva per-operatória foi utilizada para localização da perfuração gástrica.

\section{DISCUSSÃO}

A BGAVL quando comparada às demais cirurgias bariátricas possui a vantagem de ser segura e efetiva para o paciente, com baixa morbidade e tempo de hospitalização reduzido. Embora sem mortalidade cirúrgica referida, a banda gástrica vídeolaparoscópica pode evoluir com complicações per e pós-operatórias.

As séries internacionais referem três tipos de complicações: 1)- relacionadas com o port-a-cath: desconexão ou extrusão do tubo 2)- relacionadas com o ato cirúrgico: perfuração esofagiana ou gástrica, implante distópico e 3)- relacionadas com a banda: extrusão, perfuração e migração. Os processos infecciosos podem ocorrer com o port ou com a banda ${ }^{3,4}$. Também são descritas complicações clínicas decorrentes da cirurgia, destacando-se a dilatação esofagiana, que evolui com sintomas e achados endoscópicos similares a acalasia ${ }^{1}$.

Hauri et al. em seguimento de doze meses, em 207 pacientes submetidos à BGAVL, observou infecção em 4,3\%, complicações relacionadas com o port em $2,9 \%$ e, em $4,8 \%$, as complicações estavam associadas à banda gástrica, sendo $2,9 \%$, extrusão da banda. Referiu ainda, $0,5 \%$ de implante distópico e $0,5 \%$ de perfuração esofagiana ${ }^{2}$.

Wolf et al. relataram deslizamento da banda em $5 \%$ dos casos e migração com infecção em $0,7 \%{ }^{5}$.

Doldi et al. avaliaram a banda gástrica ajustável por videolaparoscopia em 109 pacientes. Como nas demais séries, não há relato de mortalidade. 
Referem dilatação da bolsa gástrica necessitando reintervenção em $5,8 \%$, perfuração gástrica peroperatória em $2,3 \%$ e migração da banda em $0,6 \%{ }^{3}$.

Angrisani et al. publicaram recentemente um estudo multi-cêntrico de 1265 pacientes submetidos à BGAVL. Nesta revisão relataram complicações relacionadas com a banda em $11,3 \%$ dos casos, com predomínio da dilatação da bolsa $(5,2 \%)$ e problemas com o port (4,2\%). A extrusão da banda foi referida em 24 pacientes $(1,9 \%)$.

Cadiere et al. observaram incidência similar em série de 652 pacientes. As complicações tardias ocorreram em $7 \%$ dos casos, com predomínio da dilatação da bolsa (25/652) e problemas com o port (18/652). A extrusão da banda foi relatada em apenas dois casos .

As pacientes examinadas neste trabalho apresentavam evolução clínica similares: ambas em pós-operatório tardio, mais de 12 meses e com manifestação clínica de dor importante, disfagia e vômitos.

A literatura mundial apresenta como opção terapêutica para a extrusão da banda a retirada cirúrgica, com ou sem conversão em outro procedimento bariátrico no mesmo ato operatório ${ }^{3,5}$. Nos dois casos apresentados neste relato, optamos por remoção cirúrgica da banda por acesso videolaparoscópico, sem conversão em outro procedimento bariátrico no mesmo ato operatório. As pacientes permanecem em controle clínico. Weiss et al. relataram, em recente publicação, a técnica de remoção da banda ajustável e do tubo, nos casos de migração, por endoscopia e retirada do port com anestesia local.

Todos os métodos intervencionistas e cirúrgicos apresentam complicações, em parte dependentes da curva de aprendizado do executor e em parte à técnica, Embora todas as séries internacionais demonstrem a segurança da BGAVL em relação às demais técnicas bariátricas, devemos estar atentos para as complicações tardias, principalmente as relacionadas com a dismotilidade esofagiana e a extrusão da banda. Esses dados são importantes, principalmente para os médicos endoscopistas que começam a examinar pacientes submetidos aos diversos tipos de cirurgia bariátrica. Durante o exame endoscópico dos pacientes submetidos à BGAVL com sintomas digestivos ou dor, o médico deve estar atento ao exame do esôfago distal, fundo e corpo gástrico. É possível, para o endoscopista experiente, avaliar a formação da "bolsa", sua posição em relação à transição epitelial esôfago-gástrica e esfíncter esofagiano inferior. Do mesmo modo, deve estar atento para o aumento do calibre do esôfago, cujo aspecto endoscópico é similar ao da acalasia. O estudo detalhado do fundo e corpo gástrico, através da retroversão, possibilita identificação adequada da compressão da banda elástica na mucosa gástrica íntegra e sua localização. O exame endoscópico cuidadoso é capaz de identificar os implantes distópicos, visualizar a banda extrusa e perfuração da mucosa.

\begin{abstract}
Various options for surgical treatment of morbid obesity have been developed with varying results: vertical banded gastroplasty with intestinal by-pass, disabsorptive surgeries and laparoscopic adjustable gastric banding. Although all of them have been effective in weight loss, lower rates of early and late postoperative complications have been described in some procedures. Laparoscopic adjustable silicone gastric banding (LASGB) has a similar principle as vertical banded gastroplasty and it is a minimally invasive procedure, with low systemic and operative problems, but not free of them. We report two rare cases of this complications of LASGB.
\end{abstract}

Key words: Videolaparoscopic adjustable gastric banding: complications; Bariatric surgery: operative complications; Morbid obesity: operative complications

\section{REFERÊNCIAS}

1. Weiss HG, Nehoda H, Labeck B et al. - Treatment of morbid obesity with laparoscopic adjustable gastric banding affects esophageal motility. Am J Surg, 2000, 180:479.

2. Hauri P, Steffen R, Ricklin T et al. - Treatment of morbid obesity with the Swedish adjustable gastric band 
(SAGB): complication rate during 12-month follow-up period. Surgery, 2000, 127: 484.

3. Doldi SB, Micheletto G, Lattuada E, et al. - Adjustable gastric band: 5-year experience. Obes Surg, 2000, 10:17.

4. Weiner R, Emmerlich V, Wagner D, et al. - Management and therapy of postoperative complications after "gastric banding" for morbid obesity. Chirurg, 1998, 69:1082.
5. Wolf AM, Kortner B, Kuhlmann HW. Results of bariatric surgery. Int J Obes Relat Metab Disord, 2001, 21:S113.

Endereço para correspondência:

Ana Maria Zuccaro

Rua Visconde de Pirajá, 414/1019

22.410-002 - Rio de Janeiro - RJ

Tel: + 5521 2522-4911 Fax: + 5521 2521-6002

E-mail: azuccaro@uninet.com.br 